\title{
ROLE OF IMPRINT CYTOLOGY IN INTRA-OPERATIVE DIAGNOSIS OF THYROID LESIONS
}

\author{
Sivaelagovan $R^{1}$, Sumathi $V^{2}$, A. Veni Alagamuthu ${ }^{3}$
}

1Assistant Professor, Department of Pathology, KAPV Government Medical College/MGM Government Hospital, Trichy, Tamilnadu, India.

${ }^{2}$ Associate Professor, Department of Pathology, KAPV Government Medical College/MGM Government Hospital, Trichy, Tamilnadu, India.

${ }^{3}$ Assistant Professor, Department of Neurology, KAPV Government Medical College/MGM Government Hospital, Trichy, Tamilnadu, India.

\section{BACKGROUND}

ABSTRACT

Intraoperative cytological diagnosis is required for the optimal extent of surgery and to know the nature of lesion, whether the lesion is malignant or not. Both Frozen Section (FS) and Touch Impression Cytology (TIC) serve this purpose well. Both provide accurate results in minutes. In this study we assess the advantages and usefulness of Touch Imprint cytological study in the diagnosis of thyroid lesions.

\section{METHODS}

A diagnostic evaluation study was conducted in the Department of Pathology, Madurai Medical College, Madurai. 752 head and neck lesions were received during the study period. Out of these, 117 were thyroid lesions. Among these thyroid lesions, 51 cases had post-surgical follow up. A correlative study between Touch Imprint Cytology and histopathology was done for these 51 cases.

\section{RESULTS}

In this study, 44 (86\%) Touch Imprint cytology reports were correlated with histopathological diagnosis. In this study, sensitivity, specificity, and accuracy is correlating well with other studies.

\section{CONCLUSIONS}

Imprint cytology is a simple, rapid and sensitive technique for the diagnosis of thyroid lesions. Touch Imprint Cytology alone may provide a correct diagnosis in the vast majority of cases with minimal expense, and without the need of sophisticated cryostat machine, thus making it quite suitable for many hospitals where cryostat machines are not available. Touch Imprint cytology has further advantage of being inexpensive, simple, and quicker than frozen section.

HOW TO CITE THIS ARTICLE: Sivaelagovan R, Sumathi V, Alagamuthu AV. Role of imprint cytology in intra-operative diagnosis of thyroid lesions. J. Evolution Med. Dent. Sci. 2019;8(14):1174-1177, DOI: 10.14260/jemds/2019/260

\section{BACKGROUND}

The largest of all the endocrine glands the Thyroid gland is unique among the endocrine glands. It is superficial in location. The thyroid gland is affected by a variety of pathological lesions like developmental, inflammatory, hyperplastic and neoplastic conditions. They are quite common in the clinical practice. Intraoperative cytological diagnosis is required for the optimal extent of surgery and to know the nature of lesion whether the lesion is malignant or not. Imprint cytology as a technique for rapid microscopical diagnosis was introduced as early as 1927.(1,2,3,4) Imprint cytology has been applied in the study of various tissues like lymph node, breast, thyroid, etc.(5) In thyroid, a correct operative diagnosis is of utmost importance especially in malignancies. While Frozen Section tissue architecture closely approximates permanent histology sections, enabling

'Financial or Other Competing Interest': None.

Submission 05-03-2019, Peer Review 25-03-2019,

Acceptance 28-03-2019, Published 08-04-2019.

Corresponding Author:

Dr. Sumathi $V$,

Associate Professor,

Department of Pathology,

KAPV Government Medical College,

MGM Government Hospital,

Trichy, Tamilnadu, India.

E-mail: lakshaelango@gmail.com

DOI: $10.14260 /$ jemds $/ 2019 / 260$

\section{(c) $($ i) $\odot$}

a degree of comfort, Touch Impression Cytology provides better, crisp cellular details and even some tissue architecture with fewer artefacts. Even when frozen section facilities are available, in cases of thyroid malignancies like papillary carcinoma where nuclear features are important for diagnosis, imprint cytology is superior to frozen sections. $(6,7,8)$

\section{Aim of the Study}

To compare the accuracy of touch imprint cytological study (cheaper) and frozen section study (expensive).

\section{METHODS}

A diagnostic evaluation study was conducted in the Department of pathology, Madurai Medical College, Madurai. A total of 752 head and neck lesions were received during the study period. Out of these, 117 were thyroid lesions. Among these thyroid lesions, 51 cases had post-surgical follow up. The detailed clinical history of these 51 patients including the duration of swelling, pain, fever, loss of weight, loss of appetite and cough with expectoration, etc. were obtained. Touch Imprint cytology was also done immediately after receiving the operated specimens. Then the results were noted, and Histopathological correlation was done for all these cases. A correlative study between Touch Imprint Cytology and histopathology was done for these 51 cases. Then all the specimens were undergone the gold standard histopathological examination. Finally, the results of Touch Imprint Cytology and the histopathology were compared. 


\section{Touch Imprint Cytology}

Touch Imprint cytology was done for 51 cases on freshly cut surface of the specimen by gently pressing the glass slide. Then the slides were immediately wet fixed in ninety five percent ethyl alcohol for five to six seconds. Then the smears were stained with haematoxylin and eosin. Results were recorded.

\section{Histopathology}

The specimens of lobectomy, hemi thyroidectomy, near total thyroidectomy and total thyroidectomy with modified neck dissection were received for histopathological examination.

The specimens were fixed in $10 \%$ formalin for $24-48$ hours. Then detailed gross examination including weight, measurement, shape, colour and consistency were noted. They were cut into parallel and longitudinal slices including the capsular invading areas. The additional features such as hemorrhage, cystic degeneration, calcification, necrosis and distance from the line of resection were noted.

The tissue slices were processed in various grades of alcohol and xylol and subsequently embedded in paraffin wax. Paraffin sections of $4 \mu \mathrm{m}$ thickness were subjected to haematoxylin and eosin staining

Touch imprint cytology, Histopathological reports of 51 cases were recorded and correlative study was done.

\section{Statistical Analysis}

All the collected information was entered in excel sheet and the analysis was done using Epidemiological Information Package (EPI). Descriptive statistics were done to express the age, sex. To assess the diagnostic ability of the FNAC in various parameters like sensitivity, specificity, positive predictive value, negative predictive value and accuracy were calculated. The following formulas were used to calculate various parameters. (Sensitivity $=$ True positive $\times 100 /$ True positive + False negative, Specificity $=$ True negative $x$ $100 /$ False positive + True negative, Positive predictive value $=$ True positive $\mathrm{x} 100 /$ True positive + False positive, Negative predictive value $=$ True negative $\mathrm{x} 100 /$ True negative + False negative, Accuracy $=$ True positive + True negative $x 100 \mathrm{~N}$ ).

\section{Ethical Issues}

To conduct the study permission was obtained from various department including Department of surgery, Department of Ear, nose and Throat, Department of surgical oncology. Informed written consent was obtained from all the study participants before doing TIC. Ethical clearance was obtained from Institutional Ethical Committee, Madurai medical College.

\section{RESULTS \\ TIC Diagnosis}

Imprint cytological diagnosis was offered for 51 cases. Out of these, 27 cases were reported as nodular goiter, 7 cases as papillary carcinoma, 6 cases as Hashimoto's thyroiditis, 2 cases as lymphocytic thyroiditis, 7 cases as follicular neoplasm, one case as Granulomatous thyroiditis and another case as Anaplastic carcinoma.

\section{Correlation Between TIC and Histopathology}

A correlative study was done between Touch imprint cytology and histopathology showed the following results. Among them 44 (86\%) Touch imprint cytology reports were correlated with histopathological diagnosis. (Chart-I)
In the present study 27 cases of Nodular goiter on Touch imprint Cytology were found to be Nodular goiter in 23 cases, Follicular adenoma in 2 cases and Papillary carcinoma in 2 cases.

2 cases of Lymphocytic thyroiditis on Touch imprint Cytology were found to be Nodular goiter in 1 case and Hashimoto's thyroiditis in one case on subsequent histopathological examination.

7 cases of Follicular neoplasm (Fig-3) on Touch imprint Cytology were found to be Follicular adenoma in 6 cases and Nodular goiter in 1 case on subsequent histopathological examination.

6 cases of Hashimoto's thyroiditis (Fig-2), 6 cases of papillary carcinoma (Fig-4), one case of Granulomatous Thyroiditis (Fig-1) and another one case of anaplastic carcinoma on Touch imprint Cytology were subsequently confirmed by histopathology.
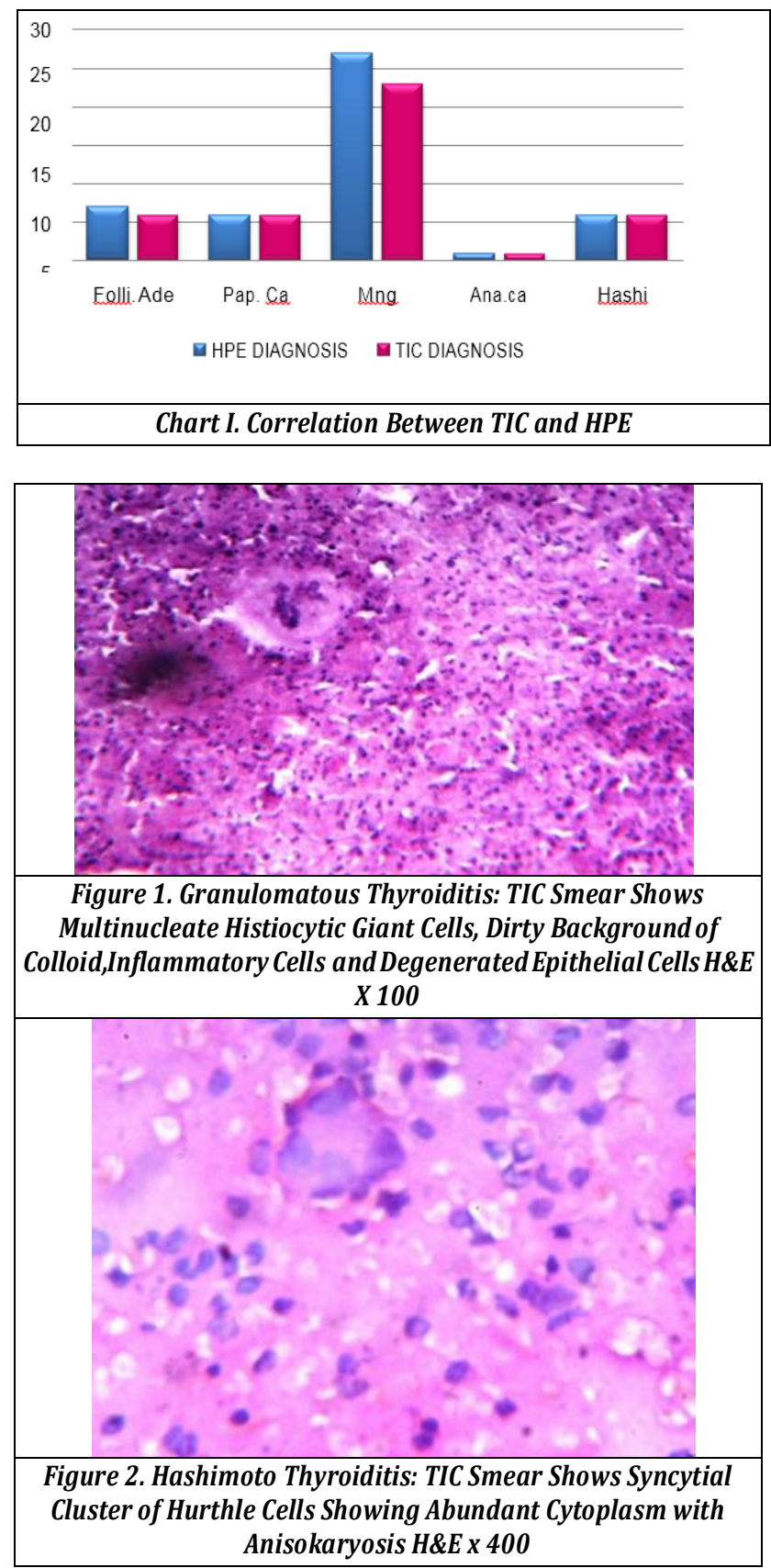

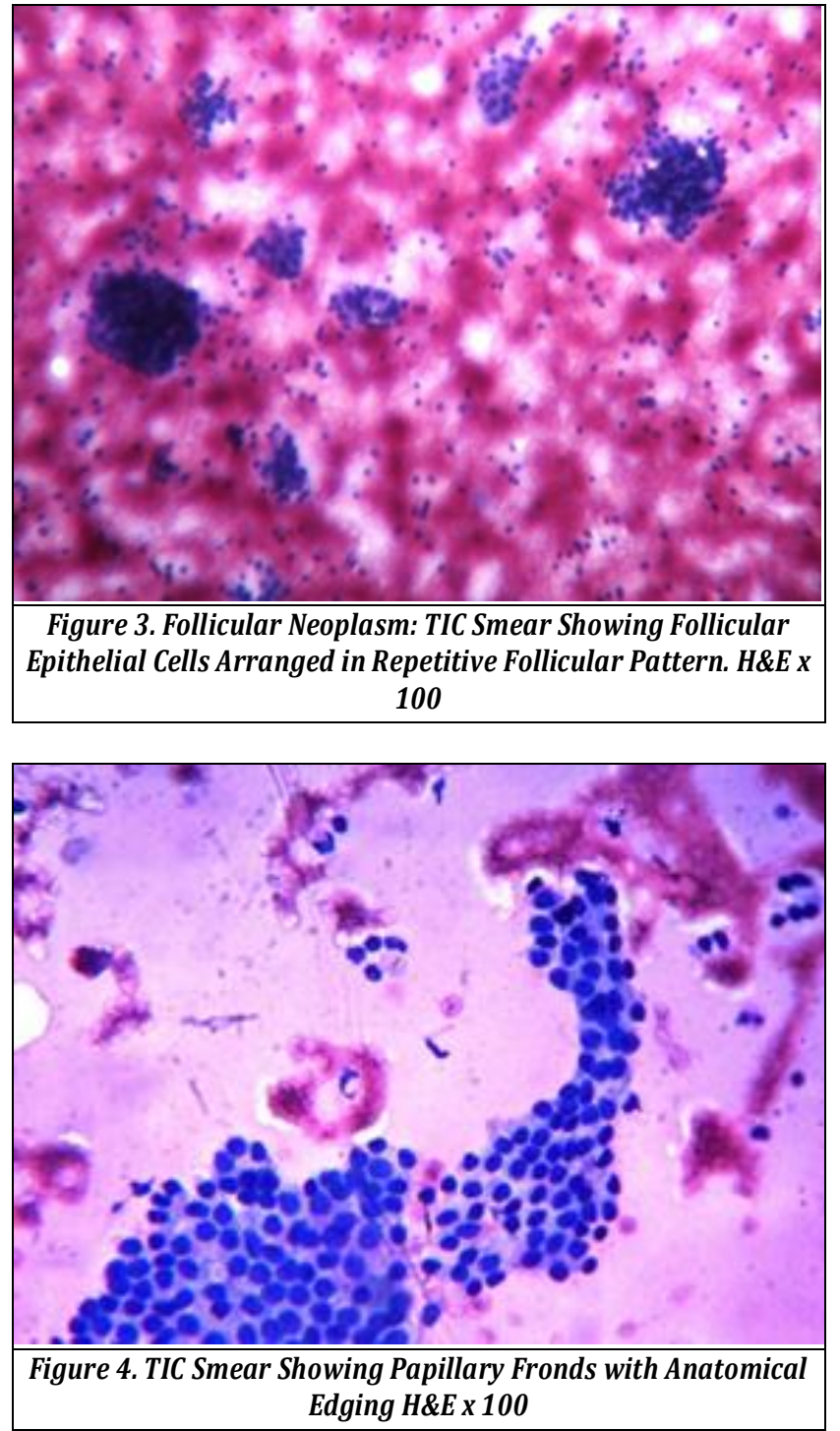

\section{DISCUSSION}

Intraoperative pathologist consultation is required by surgeons for immediate important decisions regarding the nature of the lesion and to decide the optimal extent of surgery required. Both Touch Imprint Cytology (TIC) and Frozen Section (FS) serve this purpose well. Both provide accurate results within minutes while the patient is under anesthesia. Operating Surgeon then modifies their surgical decision based on the intraoperative consultation with Pathologist. Touch Imprint Cytology provides better and crisp cellular details and even some tissue architecture.

\section{Concordance Between Touch Impression Cytology and Histopathology}

Non neoplastic lesions in Touch Impression Cytology: In the present study among the non-neoplastic lesions Nodular goiter was the most common lesion. In this study 27 cases of Nodular goiter on Touch Impression Cytology were well correlated with 23 cases in histopathology. Among the other non-neoplastic lesions 6 cases of Hashimoto's thyroiditis and 1 case of Granulomatous Thyroiditis were well correlated with histopathology. 2 cases of Lymphocytic thyroiditis on Touch Impression Cytology showed the diagnosis of Hashimoto's thyroiditis and Nodular goiter by subsequent histopathology.

\section{Neoplastic Lesions}

In the present study among the neoplastic lesions papillary carcinoma and follicular neoplasm were the most common lesions. 7 cases of papillary carcinoma, 1 case of Anaplastic carcinoma on Touch Impression Cytology were well correlated with subsequent histopathology. Among the 7 cases of follicular neoplasm 6 cases were well correlated with subsequent Histopathology. The remaining one case turned as Nodular goiter in histopathological examination.

In the present study we had problem in diagnosing 4 cases of Nodular goiter, 2 cases of Lymphocytic thyroiditis and a case of follicular neoplasm.

Concordance between Touch Impression Cytology and Histopathology is $86.27 \%$.

\section{Diagnostic Accuracy of Touch Impression Cytology}

In the present study True positive 6/51 cases, True negative 43/51 cases and False negative 2/51 cases, sensitivity, specificity, positive predictive value (PPV), negative predictive value (NPV) and diagnostic accuracy for malignant lesions of thyroid were $75 \%, 100 \%, 100 \%, 95.5 \%, 96 \%$ respectively.

The following table (Table-1) shows the comparison of sensitivity, specificity, and accuracy of Touch Impression Cytology on malignant lesions of thyroid with various authors and is correlating well.

\begin{tabular}{|c|c|c|c|}
\hline Study & Sensitivity & Specificity & $\begin{array}{c}\text { Diagnostic } \\
\text { Accuracy }\end{array}$ \\
\hline Issam et al 7 & $85 \%$ & $100 \%$ & $71.1 \%$ \\
\hline $\begin{array}{c}\text { Ahmareen } \\
\text { Khalidet al }\end{array}$ & $94.9 \%$, & $96.8 \%$. & $98.4 \%$ \\
\hline Sukumar Shaha 9 & $81.25 \%$, & $66.66 \%$, & $82.59 \%$, \\
\hline Ferit Taneri10 & $83.3 \%$ & $97.7 \%$ & $95 \%$ \\
\hline Present Study & $75 \%$ & $100 \%$ & $96 \%$ \\
\hline \multicolumn{4}{|c|}{ Table 1. Comparison of Specificity and Sensitivity for } \\
Thyroid Lesions \\
\hline
\end{tabular}

In the present study diagnostic accuracy rate, specificity by Imprint cytology is high with low sensitivity when compared to other studies. It showed good positive predictive value (PPV) and negative predictive value (NPV). Results of Frozen section by various authors shows high diagnostic accuracy rate but sensitivity was relatively lower. It pointed out very high specificity and positive predictive value $(100 \%$ in the present study), although negative predictive value (95.5\% in the present study) was less. Therefore, in this study, Touch imprint cytology (TIC) was found to be as good as frozen section in sensitivity and diagnostic accuracy.

\section{CONCLUSIONS}

Imprint cytology is a simple, rapid and sensitive technique for the diagnosis of thyroid lesions. In the present study, we feel sufficiently high accuracy rate can be achieved by imprint study, and this can be a useful guide in making decisions regarding the optimal extent of surgery required.

In spite of the advent of newer diagnostic modalities like frozen sections, imprint cytology still holds its unique position in the current perspective.

Touch Imprint Cytology alone may provide a correct diagnosis in the vast majority of cases with minimal expense 


\section{Jemds.com}

and without the need of sophisticated cryostat machine, thus making it quite suitable for many hospitals where cryostat machines are not available. Touch imprint cytology has further advantage of being inexpensive, simple and quicker than frozen section.

\section{REFERENCES}

[1] Godwin JT. Rapid cytologic diagnosis of surgical specimens. Acta Cytol 1976;20(2):111-5.

[2] Lee TK. The value of imprint cytology in tumour diagnosis. A retrospective study of 522 cases in Northern China. Acta Cytol 1982;26(2):169-71.

[3] Mavec P. Cytological diagnosis from tumour tissue using the "quick method" during operation. Acta Cytol 1967;11(3):229-30.

[4] Masuda T, Tezuka F, Konno H, et al. Intraoperative imprint cytology of the thyroid gland with computerassisted morphometric analysis of cell clusters. Anal Quant Cytol Histol 1988;10(4):294-8.

[5] Hoflen H, Weybora W. A new simple method for intraoperative fast diagnosis in thyroid surgeries. Virchows Arch A Pathol Anat Histol 1979;382:89-94.

\section{Original Research Article}

[6] Belleannee G, Verdebout J, Feoli F, et al. Role of cytology and frozen sections in the intraoperative examination of the thyroid: comparison of two experiences. Clin Exp Pathol 1999;47(6):273-7.

[7] Francis IM, Das DK. Role of Fine needle aspiration, intraoperative imprint cytology and frozen section in the diagnosis of thyroid lesions. Med Principles Pract 1999;8:173-82.

[8] Mutaharra HA, Deva W, Wanni KA, et al. Imprint cytology versus frozen section in intraoperative diagnosis of thyroid swellings. JK Practitioner 2007;8(1):27-9.

[9] Shaha S, Rahman AJEN. Comparative study of imprint cytology and frozen section in intraoperative diagnosis of thyroid lesions. Bangladesh Journal of Pathology 2009;24(1):12-5.

[10] Taneri F, Poyraz A, Salman B, et al. Using imprint cytology and frozen section in determining the surgical strategies for thyroid pathologies. Endocrine Regulations 2001;35:71-4. 